\title{
The Effects of Ecstasy on Liver Function Tests, Blood Glucose, and Lipids Profile of Male Rats
}

\author{
Mohammad Reza Shahraki ${ }^{1, *} ;$ Mahdieh Irani ${ }^{2}$ \\ ${ }^{1}$ Department of Physiology, Zahedan University of Medical Sciences, Zahedan, IR Iran \\ ${ }^{2}$ Zahedan Health Service Center, Zahedan University of Medical Sciences, Zahedan, IR Iran \\ *Corresponding author: Mohmmad Reza Shahraki, Department of Physiology, Zahedan University of Medical Sciences, Zahedan, IR Iran. Tel: +98-5413414552-5, Fax: +98-5413414563,
} E-mail:m_shahrakim@zaums.ac.ir

Received: June 10, 2014; Revised: July 20, 2014; Accepted: July 26, 2014

\begin{abstract}
Background: Ecstasy is used to improve mood and cordiality; however, based on some reports, it is neurotoxic to human users. Objectives: Because of the euphoria induced by MDMA (3,4-methylenedioxymethamphetamine) on the users, its consumption is increasing in almost all countries. This study was carried out to determine the effects of ecstasy administration in rats' blood sugar, lipids profile, and liver function tests.

Materials and Methods: The experiment was performed using 50 mature Wistar-Albino male rats. The rats were divided into five groups $(\mathrm{n}=10)$. Sham control group $(\mathrm{A})$, received tap water and ordinary rodent diet. The control (B) was administered saline but tests group C, D1, and D2 received single dose and multiple doses of MDMA, respectively. After experimental period, animals were deeply anesthetized by diethyl ether, sacrificed and the blood samples were collected for the evaluation of blood glucose, serum lipid and aspartate transaminase (AST), alanine transaminase (ALT), and alkaline phosphatase (ALK-P). Data were expressed as mean \pm SD and statistical difference was considered significant at $\mathrm{P}<0.05$.

Results: In C group, the values of blood sugar (193.8 $\pm 11.6 \mathrm{mg} / \mathrm{dL})$, low density lipoprotein (LDL) $(19.2 \pm 7.9 \mathrm{mg} / \mathrm{dL})$, and cholesterol (76.1 \pm $10.6 \mathrm{mg} / \mathrm{dL})$, were significantly increased compared with those of control A and B $(135 \pm 12.7),(140 \pm 18.8)$, and $(45.4 \pm 9.8),(49.8 \pm 2.1)(49.4 \pm$ 10.6) groups. However, aspartate transaminase (AST) and alanine transaminase (ALT) were significantly increased in groups D1 (145.8 \pm 14.7 $\mathrm{U} / \mathrm{L}),(91.1 \pm 8.1 \mathrm{U} / \mathrm{L})$, and D2 (159.4 $\pm 13.8 \mathrm{U} / \mathrm{L})$ and $(75.4 \pm 7.8)$ compared with those of group A(107.2 \pm 8.1$),(45.4 \pm 9.8), \mathrm{B}(79.8 \pm 12.1),(49.8 \pm 2.1)$, and C (115.6 \pm 17.5$),(52.1 \pm 7.6 \mathrm{U} / \mathrm{L})$. Cholesterol and LDL increased in groups C and D compared with group A.

Conclusions: These results indicated that chronic administration of MDMA affects liver as well as lipoprotein profile in male rats. The exact mechanism of action needs further investigation.
\end{abstract}

Keywords:N-Methyl-3, 4-methylenedioxyamphetamine; Alanine Transaminase; Aspartate Aminotransferases; Blood Glucose; Rats

\section{Background}

Ecstasy or 3,4-methylenedioxymethamphetamine (MDMA), one of the amphetamine components, has been widely abused as a recreational drug. Researchers have shown that ecstasy is a neurotoxic agent for dopaminergic and serotonergic neurons, lowering 5-HT transporters $(1,2)$. Moreover, they have found that MDMA side effects include hyperthermia, rhabdomyolysis, and cardiac dysrhythmias $(3,4)$. Besides, its users have linked hepatitis and liver induced injuries to the presence or absence of MDMA systemic features. Ecstasy hepatotoxicity can be identified in various levels and seems to manifest with different intensities, ranging from mild hepatitis with spontaneous resolution to fulminate liver failure, requiring liver transplantation (5, 6). The MDMA induced dopamine consumption result in releasing 5-HT striatum so that its depletion has been assessed (7). Reports carried out have observed serotonin transport and serotonergic terminal loss among ecstasy users (8).

According to Passie et al. MDMA causes significant increase in the level of plasma cortical, dehydroepiandrosterone (DHEA), and protection. However, Ecstasy, in contrast to sensual enhancement and affection emotion, hinders adverse sexual drive and functioning (9), which is associated with the probable threat of life $(10,11)$. According to Ninkovic et al. super oxide dismutase (SOD) activity increased in the liver of animals after treatment using a single dose of MDMA, while chronically treated animals exhibited increase in SOD activity after receiving the optimum dose (12). Investigations by Connor et al. showed that MDMA administration induced rapid and persistent suppression of user's immune system (13).

\section{Objectives}

Despite the status of worldwide ecstasy abuse, it is nontoxic for humans. The study was carried out to determine the effects of ecstasy tablet administration on blood sugar, lipid profile, and liver functionality tests using the male rat model. 


\section{Materials and Methods}

\subsection{Study Population}

This experimental study was conducted on 50 adult (5-7 months old) male Wistar-Albino rats. All experimental rats (weighing 200-250 g) were separately caged (one rat per cage). Animals were maintained under a standard environment at a temperature of $22 \pm 2{ }^{\circ} \mathrm{C}, 12 \mathrm{~h}$ :h light/dark cycle. Animals had a standard rat chow; food and water were provided ad-libitum. The rats were acclimatized for 5 days, then divided into five groups $(n=10)$.

\subsection{Protocol of the Experiment}

Ecstasy tablets were purchased from street narcotic vendors. The range of tablet weight was $200 \pm 2 \mathrm{mg}$. Five ecstasy tablets were analyzed in Tehran laboratory doping by GC/MS and gas chromatography (Agilent GC5973 Agilent MS 6890 USA). After analysis, percentage of the purified ecstasy in a tablet was found to be $60 \pm 2 \mathrm{mg}$. Other components of the tablets were filler, lubricant, sweetener, color blinder, and disintegrate agents. tablets had been dissolved in normal saline $0.9 \%$ before they were diluted in an appropriate dose.

Rats in sham control (A) received standard rat chow and tap water. Control (B) received saline 0.9\% (200 $\mu \mathrm{L})$ (gavages). Group $C$ was supplied with a single dose of MDMA tablet solution $(20 \mathrm{mg} / \mathrm{kg}$ ) (gavages). Eight hours prior to sacrifice, their blood samples were collected (8 hours MDMA exposure) (14). Group D1 received repeated dose of MDMA tablet solution ( $20 \mathrm{mg} / \mathrm{kg} / \mathrm{d}$ ) for 14 days. Group D1 was sacrificed 8 hours after the end of their treatment. Group D2 received repeated dose of MDMA tablets solu- tion (20 mg/kg/d gavages) for 14 days and was sacrificed 1 week after the last intake (14).

\subsection{Blood and Data Collection}

At the end of the trial, the rats were sacrificed by cervical decapitation under ether anesthesia, and blood samples were collected from the cervical vessels. Serum AST and ALT, fasting blood sugar (FBS), triglyceride (TG), and total cholesterol $(\mathrm{TCH})$ levels were measured using the standard methods adapted for RA1000 analyzer (Technicon, USA) after calibration by Pars Azmon kit Iran. Serum high density lipoprotein (HDL) was measured by precipitation of non-HDL lipoprotein with dextran $/ \mathrm{MgSO}_{4}$, followed by enzymatic cholesterol assay. Low density lipoprotein (LDL) was calculated according to Friedewald equation (15).

\subsection{Statistical Analysis}

Data were analyzed using SPSS 11.0. Analysis of variance was used to compare the treatment groups while Tukey multiple comparison test was applied to compare treatment with control groups. P values less than 0.05 were considered significant.

\section{Results}

Our results indicated that blood glucose level, increased significantly in group C and group D1 compared with other groups (Table 1). Low density lipoprotein (LDL) in group C increased significantly compared with other groups (Table 2). Serum alanine transaminase (ALT) and serum aspartate transaminase (AST) in group D1 and D2 were significantly higher than other groups (Table 1).

Table 1. Effect of Single and Multiple Dose Administration of Ecstasy on Blood Sugar and Serum AST, ALT and ALK-P in Male Rats ( $\mathrm{n}=$ 10) $\mathrm{a}, \mathrm{b}$

\begin{tabular}{lccccc}
\hline & $(\mathbf{A})$ & $(\mathbf{B})$ & $(\mathbf{C})$ & (D1) & (D2) \\
\hline $\mathbf{B S}, \mathbf{~ m g / d L}$ & $135.9 \pm 12.7$ & $140 \pm 18.8$ & $193.8 \pm 11.6$ & $176 \pm 12.3$ & $152.1 \pm 11.2$ \\
$\mathbf{A L T}, \mathbf{U} / \mathbf{L}$ & $45.4 \pm 9.8$ & $49.8 \pm 2.1$ & $52.1 \pm 7.6$ & $91.1 \pm 8.1$ & $75.4 \pm 7.8$ \\
$\mathbf{A S T}, \mathbf{U} / \mathbf{L}$ & $107.2 \pm 8.1$ & $79.8 \pm 12.1$ & $115.6 \pm 17.5$ & $145.8 \pm 14.7$ & $159.4 \pm 13.8$ \\
\hline
\end{tabular}

a Abbreviations: ALT, alanine transaminase; AST, aspartate transaminase; BS, blood sugar

$\mathrm{b}_{\mathrm{P}}<0.05$.

\begin{tabular}{|c|c|c|c|c|c|c|}
\hline & (A) & (B) & (C) & (D1) & (D2) & P Value \\
\hline LDL( $\mathrm{mg} / \mathrm{dL})$ & $9.1 \pm 4.6$ & $10.2 \pm 3.7$ & $19.2 \pm 7.9$ & $11.2 \pm 4.3$ & $8.3 \pm 5.9$ & $<0.01$ \\
\hline HDL(mg/l) & $37.6 \pm 7.7$ & $45.3 \pm 13.9$ & $42.8 \pm 3$ & $48.8 \pm 12.8$ & $46.9 \pm 8.8$ & $>0.05$ \\
\hline Chol (mg/l) & $59.1 \pm 12.1$ & $49.4 \pm 10.61$ & $61.6 \pm 7.5$ & $76.1 \pm 14.7$ & $60.1 . \pm 5.6$ & $<0.02$ \\
\hline TG ( mg/dL) & $59.1 \pm 12.1$ & $69.4 \pm 10.7$ & $71.6 \pm 7.5$ & $76.1 \pm 16.6$ & $66.5 \pm 13.3$ & $>0.05$ \\
\hline
\end{tabular}

a Abbreviations: LDL, low density lipoprotein; HDL, high density lipoprotein; Chol, cholesterol; TG, triglyceride.

$\mathrm{b}_{\mathrm{P}}<0.05$ 


\section{Discussion}

Our findings in this study pointed out that acute and repeated administration of ecstasy (MDMA), (20 mg/kg) caused a significant increase in blood sugar, serum alanine transaminase (ALT), aspartate transaminase (AST) in group D1. Our findings revealed that blood glucose increased after a single dose of MDMA administration in group $C$ and it is consistent with the findings of Graham et al. (16), that serum corticosterone and glucose increased significantly after an acute exposure to MDMA, in male and female rats. This response might be due to the effects of MDMA on hypothalamic-pituitary-adrenal (HPA) axis, which affects the serum corticosterone and glucose levels. In axis stress, catecholamine release causes epinephrine increase which leads to potent stimulatory effects on glucose production, mainly by enhancing hepatic glycogenolysis (17), resulting in blood sugar increase.

Investigation revealed that a cooperative contribution of monoamines such as dopamine and serotonin in the cyclic centre of secretion releases gonadotropins, which makes up a base for forming adaptive (sexual, feeding, and aggressive-defensive) behavior and stress reaction (18). Moreover, studies exhibited a significant prevalence of DNA injuries in sperms, tubular degeneration, and interstitial edema in male rats with chronic exposure to MDMA (19). Acute MDMA has some dominant neurohormonal effect such as increasing oxytocin, testosterone, and other hormone levels, which may facilitate psychotherapy.

On the other hand, MDMA administration is followed by a period of neurochemical recovery when low serotonin levels are often accompanied by lethargy and depression (20). Soto-Montenegro et al. (21) reported that a single dose of MDMA had hypoglycemic effects on rats, but repeated doses of MDMA did not alter their blood glucose level (22). However, our findings are not consistent with those of Soto-Montenegro et al. (21). This difference might be due to different dosages of ecstasy and their administration time. Our results are similar to those of Pourahmad et al. (23) who reported that metabolic reductive foundation of MDMA could probably induce liver toxicity through a mitochondrial/lysosomal toxic in isolated rat hepatocytes. On the other hand, our result is similar to those found by Pachmerhiwala et al. (24) that MDMA administration in rats caused an increase in blood glucose level in multiple brain regions. and the fact that this response involves both serotonergic and noradrenergic mechanisms by increased glycogenolysis.

Our study showed that repeated doses of MDMA administration caused an increase in AST and ALT value in group D1 compared with the control group. This finding is consistent with findings of Pontes et al. in 2010 (25) who reported that MDMA and ethanol are mainly metabolized in the liver with formation of toxic metabolites. In addition, our conclusion is in agreement with that of Ninkovi et al. (12) reporting that single and repeated administration of MDMA cause increased hepatic oxide- reductive status in the rat liver. Andreu et al. (5) explained that ecstasy was the most common substance targeting liver damage in patients under the age of 25. Custodio et al. (26) reported that MDMA and 4-methylthioamphetamine (MTA) induce different systemic and organ-specific effects like hepatotoxicity.

The mechanisms underlying MDMA and MTA-induced hepatotoxicity are multifactors. Beitia et al. (14) reported that ALT and AST serum activities as indicators of liver injury and their value did not alter 3 hours after a single dose of MDMA administration, but a significant increase in ALT and AST activities happened 6 hours after repeated dose administration. AST and ALT are liver enzyme markers, which indicate the health condition of these structures. Their significant increase observed in D1 and D2 compared with other groups is an indication of organ disruption (14). They also showed that repeated administration of MDMA resulted in liver injuries by increasing liver enzymes, which may lead to liver necrosis. ALK-P values in group D1, D2 and C were not significantly different to those of A and B, a major indicator of liver cholestasis. The findings, however, showed that chronic repeated dose did not induce cholestasis in the liver of treated groups of D1 and D2. Our findings pointed out that oral dose administration of ecstasy increased blood glucose and damaged liver, which results in liver necrosis. However, the mechanism behind the action is unknown yet.

\section{Acknowledgements}

We gratefully thank Deputy Research Center Zahedan University of Medical Sciences, Zahedan, Iran, and we are also grateful to Dr Soroush Dabiry for his kind cooperation.

\section{Authors' Contributions}

Shahraki and Irani developed the original idea, and protocol. They collected and analyzed the data and wrote the manuscript too.

\section{Funding/Support}

This study was financially supported by Deputy Research Center Zahedan University of Medical Sciences, Zahedan, Iran.

\section{References}

1. van Wel JH, Kuypers KP, Theunissen EL, Bosker WM, Bakker K Ramaekers JG. Effects of acute MDMA intoxication on mood and impulsivity: role of the 5-HT2 and 5-HT1 receptors. PLoS One. 2012;7(7).

2. Brncic N, Kraus I, Viskovic I, Mijandrusic-Sincic B, Vlahovic-Palcevski V.3,4-methylenedioxymethamphetamine(MDMA): an important cause of acute hepatitis. Med Sci Monit. 2006;12(11):CS107-9.

3. van Wel JH, Kuypers KP, Theunissen EL, Bosker WM, Bakker K, Ramaekers JG. Blockade of 5-HT2 receptor selectively prevents MDMA-induced verbal memory impairment. Neuropsychopharmacology. 2011;36(9):1932-9.

4. Miller RT, Lau SS, Monks TJ. Effects of intracerebroventricular administration of 5-(glutathion-S-yl)-alpha-methyldopamine on 
brain dopamine, serotonin, and norepinephrine concentrations in male Sprague-Dawley rats. Chem Res Toxicol.1996;9(2):457-65.

5. Andreu V, Mas A, Bruguera M, Salmeron JM, Moreno V, Nogue S, et al. Ecstasy: a common cause of severe acute hepatotoxicity. $J$ Hepatol.1998;29(3):394-7.

6. Ellis AJ, Wendon JA, Portmann B, Williams R. Acute liver damage and ecstasy ingestion. Gut. 1996;38(3):454-8.

7. Gudelsky GA, Yamamoto BK, Nash JF. Potentiation of 3,4-methylenedioxymethamphetamine-induced dopamine release and serotonin neurotoxicity by 5-HT2 receptor agonists. Eur J Pharmacol.1994;264(3):325-30.

8. Klugman A, Hardy S, Baldeweg T, Gruzelier J. Toxic effect of MDMA on brain serotonin neurons. Lancet. 1999;353(9160):1269-70.

9. Passie T, Hartmann U, Schneider U, Emrich HM, Kruger TH. Ecstasy (MDMA) mimics the post-orgasmic state: impairment of sexual drive and function during acute MDMA-effects may be due to increased prolactin secretion. Med Hypotheses. 2005;64(5):899-903.

10. Bernal W, Wendon J. Acute liver failure. Curr Opin Anaesthesiol 2000;13(2):113-8.

11. Mas M, Farre M, de la Torre R, Roset PN, Ortuno J, Segura J, et al Cardiovascular and neuroendocrine effects and pharmacokinetics of 3, 4-methylenedioxymethamphetamine in humans. J Pharmacol Exp Ther.1999;290(1):136-45.

12. Ninkovic M, Malicevic Z, Selakovic V, Simic I, Vasiljevic I. N-methyl-3,4-methylenedioxyamphetamine-induced hepatotoxicity in rats: oxidative stress after acute and chronic administration. Vojnosanit Pregl. 2004;61(2):125-31.

13. Connor TJ, McNamara MG, Finn D, Currid A, O'Malley M, Redmond AM, et al. Acute 3,4-methylenedioxymethamphetamine (MDMA) administration produces a rapid and sustained suppression of immune function in the rat. Immunopharmacology. 1998;38(3):253-60.

14. Beitia G, Cobreros A, Sainz L, Cenarruzabeitia E. Ecstasy-induced toxicity in rat liver. Liver. 2000;20(1):8-15.

15. Friedewald WT, Levy RI, Fredrickson DS. Estimation of the concentration of low-density lipoprotein cholesterol in plasma, without use of the preparative ultracentrifuge. Clin Chem. 1972;18(6):499-502.

16. Graham DL, Herring NR, Schaefer TL, Vorhees CV, Williams MT.
Glucose and corticosterone changes in developing and adult rats following exposure to (+/-)-3,4-methylendioxymethamphetamine or 5-methoxydiisopropyltryptamine. Neurotoxicol Teratol. 2010;32(2):152-7.

17. McCann UD, Szabo Z, Scheffel U, Dannals RF, Ricaurte GA. Posi tron emission tomographic evidence of toxic effect of MDMA ("Ecstasy") on brain serotonin neurons in human beings. Lancet. 1998;352(9138):1433-7.

18. Akhmadeev AV, Kalimullina LB. [Sex steroids and monoamines in the system of neuroendocrine regulation of amygdala functions]. Ross Fiziol Zh Im I M Sechenova. 2011;97(5):483-91.

19. Barenys M, Macia N, Camps L, de Lapuente J, Gomez-Catalan J, Gonzalez-Linares J, et al. Chronic exposure to MDMA (ecstasy) increases DNA damage in sperm and alters testes histopathology in male rats. Toxicol Lett. 2009;191(1):40-6.

20. Parrott AC. The potential dangers of using MDMA for psychotherapy. J Psychoactive Drugs. 2014;46(1):37-43.

21. Soto-Montenegro ML, Vaquero JJ, Arango C, Ricaurte G, GarciaBarreno P, Desco M. Effects of MDMA on blood glucose levels and brain glucose metabolism. Eur J Nucl Med Mol Imaging. 2007;34(6):916-25.

22. Greer G, Strassman RJ. Information on "Ecstasy". Am J Psychiatry. 1985;142(11):1391.

23. Pourahmad J, Eskandari MR, Nosrati M, Kobarfard F, Khajeamiri AR. Involvement of mitochondrial/lysosomal toxic cross-talk in ecstasy induced liver toxicity under hyperthermic condition. Eur JPharmacol. 2010;643(2-3):162-9.

24. Pachmerhiwala R, Bhide N, Straiko M, Gudelsky GA. Role of serotonin and/or norepinephrine in the MDMA-induced increase in extracellular glucose and glycogenolysis in the rat brain. Eur $J$ Pharmacol. 2010;644(1-3):67-72.

25. Pontes H, de Pinho PG, Fernandes E, Branco PS, Ferreira LM, Car$\mathrm{mo} \mathrm{H}$, et al. Metabolic interactions between ethanol and MDMA in primary cultured rat hepatocytes. Toxicology. 2010;270(23):150-7.

26. Custodio JB, Santos MS, Goncalves DI, Moreno AJ, Fernandes E, Bastos ML, et al. Comparative effects of 3,4-methylenedioxymethamphetamine and 4-methylthioamphetamine on rat liver mitochondrial function. Toxicology. 2010;270(2-3):99-105. 